\title{
Musashi-2 is a prognostic marker for the survival of patients with cervical cancer
}

\author{
YAQIONG LIU ${ }^{1,2}$, YI FAN ${ }^{1,2}$, XIAOYU WANG ${ }^{1}$, ZIJIAN HUANG ${ }^{2}, \mathrm{KUN} \mathrm{SHI}^{2}$ and BEI ZHOU ${ }^{2}$ \\ ${ }^{1}$ Department of Obstetrics and Gynecology, The First Affiliated Hospital of Jinan University, \\ Guangzhou, Guangdong 510630; ${ }^{2}$ Department of Obstetrics and Gynecology, Guangzhou Women and \\ Children's Medical Center, Guangzhou Medical University, Guangzhou, Guangdong 510000, P.R. China
}

Received July 12, 2016; Accepted September 7, 2017

DOI: $10.3892 / 01.2018 .8077$

\begin{abstract}
Cervical cancer is one of the most common gynecological malignancies. Mousasi 2 (Msi2) is a RNA-binding protein that regulates various key cellular functions and has emerged as a crucial regulator of cancer development. However, the clinical significance and biological functions of Msi2 in cervical cancer remain unknown. The current study assessed the expression of Msi2 mRNA using reverse transcription-quantitative polymerase chain reaction. Furthermore, the expression of Msi2 was examined in 162 cervical cancer samples using immunohistochemistry and the association between Msi2 expression and patient clinicopathological features was analyzed. The overall survival (OS) and progression-free survival (PFS) of patients were estimated using the Kaplan-Meier method and Cox regression analysis was performed to investigate the clinicopathological significance of Msi2 expression. In vitro migration and invasion assays were performed in Sinha and Caskie cells. The results demonstrated that, compared with normal cervical tissues, the expression of Msi2 was increased in cervical cancer tissues. The expression of Msi2 was significantly correlated with International Federation of Gynaecology and Obstetrics (FIGO) stage $(\mathrm{P}=0.049)$ and lymph node metastasis $(\mathrm{P}=0.036)$. Furthermore, patients with higher Msi2 expression exhibited significantly poorer OS $(\mathrm{P}=0.013)$ and PFS $(\mathrm{P}=0.006)$ than patients with low Msi2 expression. Notably, high Msi2 expression was correlated with poorer OS in patients with a FIGO stage $\leq \mathrm{I}(\mathrm{P}=0.015)$, a smaller tumor size $(\mathrm{P}=0.043)$ and grade 3 tumor $(\mathrm{P}=0.002)$. High Msi2 expression was also correlated with a poorer PFS in patients with a FIGO stage $\leq \mathrm{I}(\mathrm{P}=0.016)$ and grade 3 tumor $(\mathrm{P}=0.001)$. Multivariate analysis suggested that Msi2 expression was an independent prognostic marker of the OS $(\mathrm{P}=0.027)$ and PFS $(\mathrm{P}=0.013)$ of patients with cervical
\end{abstract}

Correspondence to: Dr Xiaoyu Wang, Department of Obstetrics and Gynecology, The First Affiliated Hospital of Jinan University, 613 E Huangpu Avenue, Guangzhou, Guangdong 510630, P.R. China E-mail:wxygynecol@126.com

Key words: Musashi-2, cervical cancer, migration, invasion, prognosis cancer. Furthermore, Msi2 knockdown significantly $(\mathrm{P}<0.05)$ inhibited the invasion and migration of cervical cancer cells. The results of the current study demonstrate that Msi2 may act as a prognostic biomarker in patients with cervical cancer. Targeting Msi2 may therefore offer a promising therapeutic strategy for the treatment of patients with cervical cancer.

\section{Introduction}

Cervical cancer is one of the most common causes of cancer-associated mortality among patients with gynecological malignancies and $\sim 85 \%$ of all cervical cancer cases occur in less developed countries (1). Despite advances in diagnosis and management, the clinical prognosis of patients with advanced cervical cancer remains poor, due to early lymph node metastasis and the 5-year survival rate is $<30 \%$ (2). Currently, certain clinicopathological variables, including tumor size and parametrical infiltration, are used to predict the risk of tumor progression, however they are insufficient at accurately predicting cancer metastasis and survival (3). Thus, it is important to identify novel biomarkers for the early detection of cancer cell metastasis, prediction of patient prognosis and individualization of treatment.

It is currently accepted that RNA-binding proteins (RBPs) serve an important role in cancer development. Such proteins act as RNA splicing factors and drive post-transcriptional gene expression programs specific to a particular cell state (4). RBPs have also been implicated in cell state transitions in the stem cell and cancer biology fields (5). The Mousasi (MS) family comprises some of the most highly conserved RBPs and in mammals, two members of this family, Msi1 and Msi2, have been identified to date (6). MS genes are expressed in stem cells and it has been suggested that they contribute to stemless maintenance in adults and to the development of organs via the translational regulation of their mRNA ligands (7). Msi2 is a highly conserved protein harboring two tandem RNA recognition motifs and a carboxyl-terminal poly-A-binding protein association domain. It specifically targets developmental transcriptional factors and cell cycle regulators (8). Msi2 is preferentially expressed in hematopoietic stem cells (HSCs), where it is required for HSC repopulation and its overexpression increases long-term engraftment of HSCs (9). Initially, Msi2 was identified as a fusion partner of homeobox protein 
HOX-A9 in the accelerated phase of chronic myeloid leukemia or during blast crises (10). It was then identified that HOX-A9 positively regulates the proliferation of HSCs and inhibits their differentiation into mature blood cells, thus promoting leukemogenic (11). Furthermore, Msi2 expression is correlated with solid tumors. Wang et al (11) demonstrated that Msi2 promotes tumor cell migration and invasion in hepatitis B virus-related hepatocellular carcinoma (HCC) cells. Msi2 depletion leads to decreased HCC migration and invasion, and the dysregulated expression of $\beta$-catenin, $\mathrm{T}$ cell factor and lymphoid enhancer factor (12). Kudinov et al (13) reported that Msi2 provides essential support for tumor growth factor $\beta 1 /$ mothers against decapentaplegic homolog 3 signaling and contributes to non-small cell lung cancer metastasis. However, to the best of our knowledge, the contribution of Msi2 to cervical carcinoma has not yet been elucidated.

In the present study, the expression and prognostic value of Msi2 in patients with cervical cancer was determined. It was demonstrated that Msi2 is highly expressed in human cervical cancer tissues and is correlated with tumor stage and lymph node metastasis. In addition, Msi2 represents an independent prognostic factor for overall survival (OS) and progression-free survival (PFS) in patients with cervical cancer. Furthermore, Msi2 promotes the migration and invasion of cervical cancer cells. Taken together, the results of the current study suggest that Msi2 serves an important role in the development and progression of cervical cancer and may be a useful prognostic marker in patients with cervical cancer.

\section{Patients and methods}

Cell Lines. The cervical cancer cell lines Sinha and Caskie were purchased from the Type Culture Collection of the Chinese Academy of Sciences (Shanghai, China). Each cell line was cultured in Dulbecco's Modified Eagle medium (DMEM; Invitrogen; Thermo Fisher Scientific, Inc., Waltham, MA, USA). The medium was supplemented with $10 \%$ fetal bovine serum (HyClone; GE Healthcare, Logan, UT, USA) and $1 \%$ penicillin-streptomycin (Invitrogen; Thermo Fisher Scientific, Inc.). Cells were cultured in a $5 \% \mathrm{CO}_{2}$-humidified incubator at $37^{\circ} \mathrm{C}$ until experiments were performed.

Patients. A total of 162 female patients with cervical cancer who were diagnosed and treated at The First Affiliated Hospital of Jinan University (Guangzhou, China) between January 2003 and December 2007, were retrospectively enrolled in the current study. The mean age of the patients was 44.9 years. Archived paraffin-embedded cervical cancer tissue specimens were retrieved, and clinicopathological characteristics of these patients were retrieved from the pathology file of The First Affiliated Hospital of Jinan University. A further 6 pairs of snap-frozen samples of cervical cancer and adjacent non-cancerous tissues were collected from The First Affiliated Hospital of Jinan University between July 2015 and December 2015, and stored in liquid nitrogen at $-196^{\circ} \mathrm{C}$ prior to reverse transcription-quantitative polymerase chain reaction (RT-qPCR). The mean age of the 6 patients was 43 years. To obtain tumor adjacent non-cancerous tissues, grossly normal cervical tissues $5 \mathrm{~cm}$ away from the resection margin were carefully dissected and subjected to freezing and evaluation to exclude cancerous or inflammatory cells. None of the patients had received preoperative chemotherapy or radiation treatment. Diagnoses and grading were determined following the latest International Federation of Gynecology and Obstetrics criteria (FIGO) (14). Prior written patient consent was obtained and the Research Ethics Committee of The First Affiliated Hospital of Jinan University approved the present study.

$R T-q P C R$. The expression of Msi2 in cervical cancer and paired adjacent non-cancerous cervical tissues was determined by RT-qPCR. Total RNA was extracted from tissues using TRIzol reagent (Invitrogen; Thermo Fisher Scientific, Inc.) following the manufacturer's protocol. Extracted RNA was pretreated with RNase-free DNase and $2 \mu \mathrm{g}$ RNA from each sample was used for cDNA synthesis with random hexamers using cDNA Synthesis kit (Thermo Fisher Scientific, Inc.), according to manufacturer's protocol. qPCR was performed using a SYBR green PCR kit (Toyobo Life Science, Osaka, Japan) on an ABI 7500 Real-Time PCR system (Applied Biosystems; Thermo Fisher Scientific, Inc.). The thermocycling conditions were as follows: Pre-degeneration at $95^{\circ} \mathrm{C}$ for $5 \mathrm{~min}$, degeneration at $95^{\circ} \mathrm{C}$ for $5 \mathrm{sec}$ and annealing at $60^{\circ} \mathrm{C}$ for $30 \mathrm{sec}$ (40 cycles). The primers used were as follows: Msi2, forward, 5'-AGC ACGACCCCGGTAAAATG-3', and reverse, 5'-GCTCGA CGAGGAAATGCAAC-3' and GAPDH, forward, 5'-CGA GATCCCTCCAAAATCAA-3', and reverse, 5'-TTCACACCC ATGACGAACAT-3'. For calculation of relative quantification, the $2-\Delta \Delta \mathrm{Cq}$ formula was used, where $-\Delta \Delta \mathrm{Cq}=(\mathrm{Cq}$, target $-\mathrm{Cq}$, GAPDH) experimental sample-(Cq, target-Cq, GAPDH) control sample (15).

Transfection with small interfering (si) RNA. siRNAs targeting Msi2 and control siRNA were synthesized and purified by Guangzhou Ribobio, Inc. (Guangzhou, Guangdong, China). The siRNA sequences used were as follows: RNAi\#1: 5'-CAA UGCUGAUGUUUGAUAAdTdT-3' and RNAi\#2: 5'-GGA GAAAGUCUGUGAGAUUdTdT-3'. The control siRNA sequence was: ACGUGACACGUUCGGAGAA. Transfection was performed using Lipofectamine ${ }^{\circledR} 2000$ Transfection reagent (Invitrogen; Thermo Fisher Scientific, Inc.) following the manufacturer's protocols. Final concentrations of all siRNAs were $100 \mathrm{nM}$ and cells were harvested $72 \mathrm{~h}$ following transfection.

Immunohistochemistry. Paraffin-embedded archival specimens were cut into sections $4-\mu \mathrm{m}$ thick and baked at $65^{\circ} \mathrm{C}$ for $30 \mathrm{~min}$. Sections were then dewaxed in xylene and rehydrated in grade alcohol, followed by submerging in citrate antigenic retrieval buffer ( $\mathrm{pH}$ 6.0). Following inhibition of endogenous peroxidase activity by $3 \%$ hydrogen peroxide in methanol, slides were incubated with $1 \%$ bovine serum albumin at $25^{\circ} \mathrm{C}$ for $30 \mathrm{~min}$. (Gibco; Thermo Fisher Scientific, Inc.) to block non-specific binding. Subsequently, tissue sections were incubated with anti-Msi2 antibody (1:200; cat. no. ab76148; Abcam, Cambridge, MA, USA) overnight at $4^{\circ} \mathrm{C}$. Following washing, slides were incubated with the biotinylated goat anti-rabbit secondary antibody (1:1,000; cat. no. ab7090; Abcam) at $25^{\circ} \mathrm{C}$ for $45 \mathrm{~min}$, followed by further incubation with streptavidin-horseradish peroxidase complex. Finally, 
A

Cervical cancer

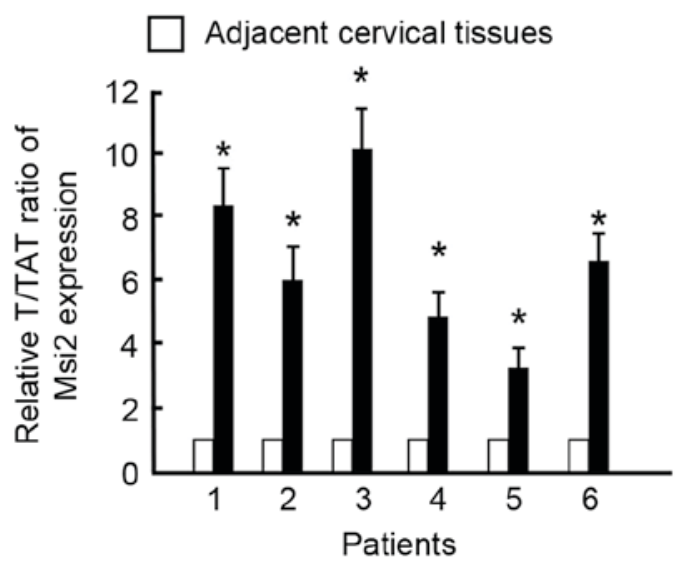

B
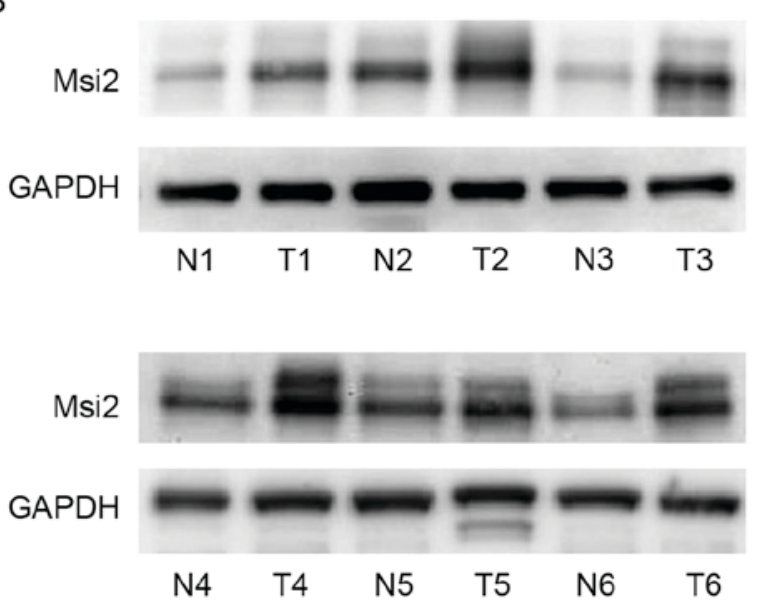

Figure 1. Expression of Msi2 mRNA in 6 pairs of cervical cancer tissues and adjacent non-tumor tissues analyzed by (A) reverse transcription-quantitative polymerase chain reaction and (B) western blotting. Bars represent the means of three independent experiments. "P<0.05 vs. adjacent cervical tissues. Msi2, Musashi-2; T, tumor; N, normal; T/TAT, tumor/tumor adjacent tissue.

A

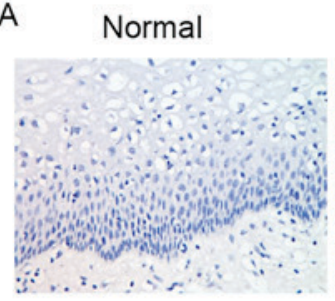

Stage la

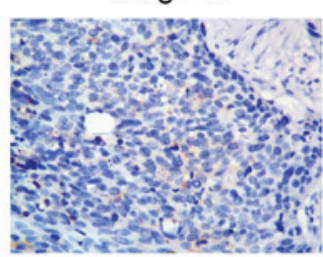

Stage Ib

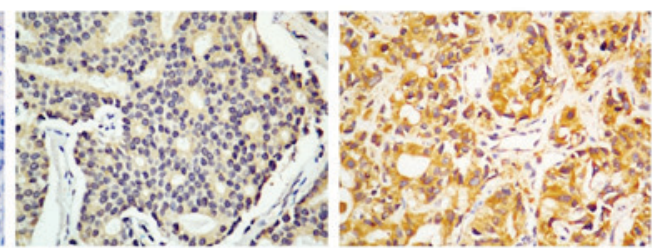

Stage Ilb

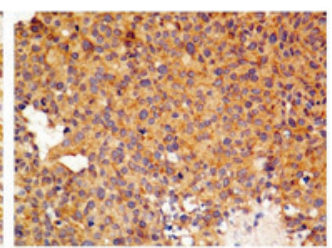

B

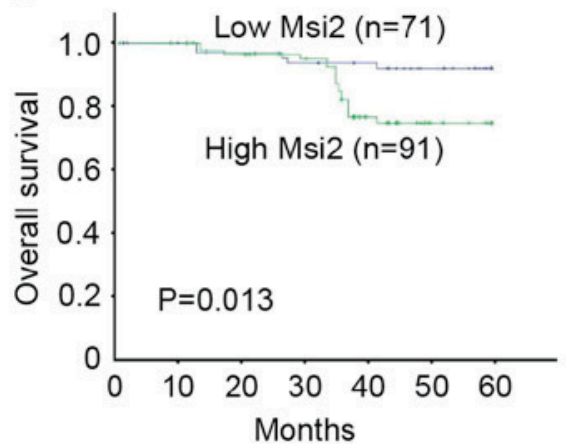

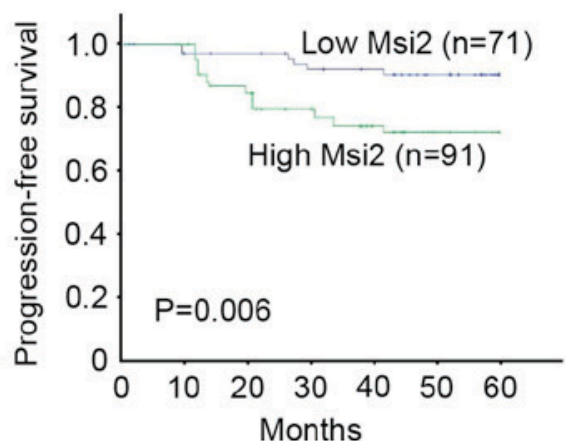

C

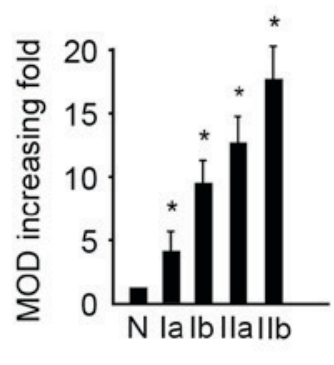

Figure 2. Msi2 expression is associated with tumor stage and the clinical prognosis of patients with cervical cancer. (A) Immunohistochemical staining of Msi2 expression in cervical cancer (FIGO stages Ia-II) and normal cervical tissues (magnification, x400). Msi2 expression was undetectable in normal cervical tissues; however, it was increased in stage Ia-stage II cervical cancer tissues. (B) Kaplan-Meier survival curves comparing cumulative overall and progression-free survival rates between patients with low and high expression of Msi2. (C) Quantification of the average MOD of Msi2 staining between normal cervical tissue and cervical cancer tissues at different clinical stages. ${ }^{*} \mathrm{P}<0.05$ vs. N group. Msi2, Musashi-2; MOD, mean optical density; FIGO, International Federation of Gynecology and Obstetrics.

the sections were counterstained with hematoxylin at $25^{\circ} \mathrm{C}$ for 5 min and mounted under cover-slips.

The degree of immunostaining was independently reviewed and scored by two observers using a transmitted light microscope (magnification, $\mathrm{x} 40$ ) based on the proportion of positively stained tumor cells and the intensity of staining. The proportion of stained cells was evaluated and recorded as: 0 , no staining; $1,<10 \%$ of tumor cells exhibiting positive staining; $2,10-50 \%$ of tumor cells exhibiting positive staining; $3,51-80 \%$ of tumor cells exhibiting positive staining; and 4 ,
$>80 \%$ of tumor cells exhibiting positive staining. The intensity of staining was graded semi-quantitatively as follows: 0, no staining; 1, weak staining; 2, positive staining and 3, strong staining. The staining index (SI) was calculated by multiplying the staining intensity score and the proportion of stained tumor cells. A SI of $\leq 4$ was defined as low Msi2 expression, whereas a SI of $>4$ was defined as high Msi2 expression.

Western blot analysis. Western blot analysis was performed following standard procedures. Cells and tissue samples 
were lysed in RIPA lysis buffer (BiYunTian, Shanghai, China), and the protein concentrations was detected using a BCA Protein Assay kit (Thermo Fisher Scientific, Inc.). Total proteins (30 $\mu \mathrm{g} / \mathrm{lane})$ were loaded onto each well by SDS-PAGE using $10 \%$ gels and transferred to a polyvinylidene difluoride (PVDF) membrane. The blots were blocked in $5 \%$ milk for $1 \mathrm{~h}$ at room temperature. PVDF membranes were incubated with the primary monoclonal rabbit anti-Msi2 (1:1,000; cat. no. ab76148; Abcam) antibodies overnight at $4^{\circ} \mathrm{C}$. Subsequently, membranes were incubated with corresponding goat anti-rabbit secondary antibodies $(1: 5,000$; cat. no. ab6721; Abcam) for $2 \mathrm{~h}$ at room temperature and detected by chemiluminescence. Proteins were visualized using an enhanced chemiluminescence reagent (ECL Prime, GE Healthcare). GAPDH (1:1,000; cat. no. ab9484; Abcam) was used as a loading control.

Wound healing assay. A total of $5 \times 10^{5}$ cells per well were seeded in 6-well plates, then transfected with Msi2 siRNA following overnight incubation. When cells reached $90 \%$ confluence, the cell monolayer was scratched with a sterile pipette tip, floating cells were removed with PBS and cells were cultured in DMEM at room temperature for $24 \mathrm{~h}$. Photographs were taken at 0 and $24 \mathrm{~h}$ along the scrape line using a light microscope (magnification, $\mathrm{x} 20$ ). The results are expressed as the relative wound closure distance. The experiment was conducted in triplicate.

Cell migration and invasion assay. The migration ability of Caskie and Sinha cells transected with Msi-2-siRNA was measured in 24-well Transwell chambers (BD Biosciences, Franklin Lakes, NJ, USA). For the migration assay, cells $\left(1 \times 10^{5}\right.$ cells) in DMEM were plated in the upper chamber and incubated at $37^{\circ} \mathrm{C}$ in humidified air containing $5 \% \mathrm{CO}_{2}$, and allowed to invade towards DMEM containing 10\% FBS (HyClone; GE Healthcare) in the lower chamber. Following $24 \mathrm{~h}$ incubation, the upper surface of the membrane was gently wiped to remove non-migrating cells. Cells that migrated to the underside were fixed in $4 \%$ paraformaldehyde at $0^{\circ} \mathrm{C}$ for $20 \mathrm{~min}$, stained with $0.1 \%$ crystal violet for $15 \mathrm{~min}$ at $25^{\circ} \mathrm{C}$ and counted in five random fields using a light microscope (magnification, x20). For cell invasion experiments, Matrigel (BD Biosciences) was plated inside Transwell culture inserts, with the same protocol being followed.

Statistical analysis. All statistical analyses were performed using the SPSS 16.0 statistical software package (SPSS, Inc., Chicago, IL, USA). Experiments were performed in triplicate and differences between data were analyzed using one-way analysis of variance followed by Dunnett's test. The association between Msi2 expression and patient clinicopathological characteristics was analyzed using the $\chi^{2}$ test. Survival curves were plotted using the Kaplan-Meier method and compared using the log-rank test. Multivariate statistical analysis was performed using a Cox regression model. $\mathrm{P}<0.05$ was considered to indicate a statistically significant difference.

\section{Results}

Msi2 expression is upregulated in cervical cancer. To investigate the role of Msi2 during the progression of cervical
Table I. Association between Msi2 expression and patient clinicopathological characteristics.

\begin{tabular}{|c|c|c|c|c|}
\hline \multirow[b]{2}{*}{ Parameters } & \multirow[b]{2}{*}{$\mathrm{n}$} & \multicolumn{2}{|c|}{$\begin{array}{c}\text { Msi2 } \\
\text { expression }\end{array}$} & \multirow[b]{2}{*}{ P-value } \\
\hline & & Low & High & \\
\hline Age, years & & & & 0.452 \\
\hline$\leq 50$ & 109 & 50 & 59 & \\
\hline$>50$ & 53 & 21 & 32 & \\
\hline SCC level, ng/ml & & & & 0.981 \\
\hline$\leq 1.5$ & 40 & 15 & 25 & \\
\hline$>1.5$ & 122 & 46 & 76 & \\
\hline FIGO stage & & & & $0.049^{\mathrm{a}}$ \\
\hline$\leq \mathrm{I}$ & 125 & 60 & 65 & \\
\hline$>I$ & 37 & 11 & 26 & \\
\hline Tumor size, cm & & & & 0.474 \\
\hline$\leq 4$ & 112 & 47 & 65 & \\
\hline$>4$ & 50 & 24 & 26 & \\
\hline Differentiation & & & & 0.337 \\
\hline $1 / 2$ & 64 & 25 & 39 & \\
\hline 3 & 98 & 46 & 53 & \\
\hline Histological type & & & & 0.344 \\
\hline SCC & 133 & 56 & 77 & \\
\hline $\mathrm{AC}$ & 29 & 15 & 14 & \\
\hline LN Metastasis & & & & $0.004^{\mathrm{a}}$ \\
\hline No & 132 & 62 & 70 & \\
\hline Yes & 30 & 9 & 21 & \\
\hline
\end{tabular}

${ }^{\mathrm{a}} \mathrm{P}<0.05$; Msi2, Musashi-2; SCC, squamous cell cancer; FIGO, International Federation of Gynecology and Obstetrics; AC, adenocarcinoma; LN, lymph node; y, years.

cancer, the current study examined Msi2 expression in 6 pairs of frozen cervical and adjacent non-tumor tissues using RT-qPCR. Levels of Msi2 mRNA were significantly upregulated in all cervical cancer samples compared with paired non-tumor tissues $(\mathrm{P}<0.05$; Fig. $1 \mathrm{~A})$. Western blotting also revealed similar results regarding levels of protein (Fig. 1B) in the 6 paired samples, confirming that the Msi2 is overexpressed in cervical cancer tissues.

Msi2 expression is associated with the clinicopathological features and survival of patients with cervical cancer. To investigate the association between Msi2 expression and the clinical features of cervical cancer, Msi2 expression was examined in a cohort of 162 archived cervical cancer tissues (Fig. 2). The results demonstrated that Msi2 expression was upregulated in cervical cancer tissues and was increased further in patients with advanced tumor tissue (Fig. 2A). Furthermore, quantitative analysis indicated that the mean optical density (MOD) of Msi2 staining in FIGO stage Ia-IIb primary tumors were significantly higher than those in normal cervical tissues $(\mathrm{P}<0.01$; Fig. $2 \mathrm{C})$. It was demonstrated that Msi2 expression was significantly associated with FIGO stage 
Table II. Multivariate Cox regression analyses of OS and PFS for patients with cervical cancer.

\begin{tabular}{|c|c|c|c|c|}
\hline \multirow[b]{2}{*}{ Prognostic variables } & \multicolumn{2}{|l|}{ OS } & \multicolumn{2}{|l|}{ PFS } \\
\hline & HR $(95 \% \mathrm{CI})$ & P-value & $\mathrm{HR}(95 \% \mathrm{CI})$ & P-value \\
\hline Age (>50 vs. $\leq 50$ years) & $2.272(0.820-6.297)$ & 0.115 & $2.273(0.888-5.817)$ & $0.087^{\mathrm{a}}$ \\
\hline SCC level (>1.5 vs. $\leq 1.5 \mathrm{ng} / \mathrm{ml})$ & $1.624(0.163-4.673)$ & 0.542 & $1.984(0.354-4.763)$ & 0.733 \\
\hline FIGO Stage (>I vs. $\leq \mathrm{I})$ & $1.506(0.590-3.846)$ & 0.392 & $1.258(0.535-2.960)$ & 0.599 \\
\hline Tumor size ( $>4$ vs. $\leq 4 \mathrm{~cm}$ ) & $1.258(0.509-3.109)$ & 0.619 & $1.090(0.456-2.607)$ & 0.846 \\
\hline Differentiation (Grade 3 vs. 1/2) & $1.045(0.416-2.621)$ & 0.926 & $1.516(0.633-3.630)$ & 0.350 \\
\hline Histological type (SCC vs. AC) & $2.906(1.013-8.339)$ & 0.057 & $2.129(0.782-5.792)$ & 0.139 \\
\hline LN Metastasis (+ vs. -) & $4.078(1.755-9.474)$ & 0.001 & $3.454(1.579-7.559)$ & $0.002^{\mathrm{a}}$ \\
\hline Msi2 expression (high vs. low) & $3.105(1.139-8.465)$ & 0.027 & $3.181(1.274-7.941)$ & $0.013^{\mathrm{a}}$ \\
\hline
\end{tabular}

${ }^{a} \mathrm{P}<0.05$; OS, overall survival; PFS, progression-free survival; HR, hazard ratio; CI, confidence interval; Msi2, Mousasi 2; SCC, squamous cell carcinoma; FIGO, International Federation of Gynecology and Obstetrics; AC, adenocarcinoma; LN, lymph node.

$(\mathrm{P}=0.049)$ and lymph node status $(\mathrm{P}=0.036)$. However, it was not associated with age, SCC level, tumor size, tumor differentiation or histological type (Table I).

Msi2 expression is associated with the prognosis of patients with cervical cancer. The prognostic significance of Msi2 in cervical cancer was determined using Kaplan-Meier survival analysis. The results demonstrated that the OS $(\mathrm{P}=0.013)$ and PFS $(\mathrm{P}=0.006)$ were significantly decreased in patients with higher Msi2 expression (SI >4) compared with patients with lower Msi2 expression (SI $\leq 4$; Fig. 2B). Multivariate Cox proportional hazards model analysis demonstrated that Msi2 expression was an independent risk factor for the OS $(\mathrm{P}=0.027)$ and PFS (0.013) of patients with cervical cancer (Table II). The prognostic value of Msi2 expression in selected patient subgroups was analyzed further, following the stratification of patients according to age, histological type, FIGO stage, tumor size, tumor differentiation or lymph node status (Fig. 3). Patients with tumors exhibiting high Msi2 expression had a significantly shorter OS than patients with tumors exhibiting low levels of Msi2 expression in the FIGO stage $\leq \mathrm{I}$ subgroup $(\mathrm{P}=0.015$; Fig. $3 \mathrm{~A})$ and the grade 3 subgroup $(\mathrm{P}=0.002$; Fig. 3E). By contrast, no significant differences in OS were observed between tumors exhibiting high or low Msi2 expression in the subgroups of patients with tumor size $<4 \mathrm{~cm}$ ( $\mathrm{P}=0.078$; Fig. 3C), advanced carcinomas (FIGO stage $>$ I), grade $1 / 2$ tumors, age, histological type or lymph node status (data not shown). However, high Msi2 expression and PFS were significantly associated in patients with early stage $(\mathrm{P}=0.016$; Fig. 3B), tumor size $<4 \mathrm{~cm}(\mathrm{P}=0.043$; Fig. 3D) and tumor grade $3(\mathrm{P}=0.001$; Fig. $3 \mathrm{~F})$. There were no significant differences with respect to PFS in the subgroups of patients with FIGO stage $>$ I, large tumors, grade 1/2 tumors, age, histological type, or lymph node status (data not shown).

Msi2 knockdown suppresses the migration and invasion of cervical cancer cells. To investigate the role of Msi2 in the migration and invasion of cervical cancer cells, Msi2 expression was silenced using specific siRNAs (Fig. 4A). As expected, following Msi2-knockdown, cells exhibited a significantly slower closure of the wound area than control cells $(\mathrm{P}<0.05$; Fig. 4B) and the migration and invasion potentials of Caskie and Sinha cells were significantly reduced compared with their respective control cells (all $\mathrm{P}<0.05$; Fig. 4C). These results suggest that Msi2 serves an important role in the migration and invasion of cervical cancer cells.

\section{Discussion}

Despite advances in the therapeutic strategies available to treat cervical cancer, the long-term prognosis of patients with recurrent and metastatic cervical cancer remains unsatisfactory (2). Cancer cell invasion is a key factor affecting the survival of these patients (2); thus, it is important to identify novel biomarkers of cervical cancer progression and prognosis. The present study evaluated and quantified the expression of Msi2 in a set of paired cervical cancer tissues and 162 archived paraffin-embedded cervical cancer samples. It was demonstrated that Msi2 expression is upregulated in cervical cancer tissues compared with normal cervical tissues. Msi2 expression was correlated with FIGO stage, lymph node metastasis and the clinical prognosis of patients with cervical cancer. Furthermore, downregulation of Msi2 inhibited cervical cancer cell migration and invasion. Taken together, the results of the current study indicated that Msi2 may be a potential predictive factor of cervical cancer metastasis and patient prognosis.

Mousasi proteins are an evolutionarily conserved family of RBP proteins that serve a critical role in stem cells. Msi1 is expressed in neural progenitor and stem cells and helps to maintain stem cell status during the development of the central nervous system (16). Msi2 is expressed predominantly in haematopoietic stem and progenitor cells and regulates the proliferation and differentiation of progenitor cells. During leukemogenic, the downregulation of Msi2 is associated with the decreased expression of genes associated with cell proliferation and survival, as well as the elevated expression of markers of myeloid differentiation (10). However, few studies have investigated the expression of Msi2 expression in solid tumors. Using immunohistochemistry, Moreira et al (17) detected that the expression of Msi2 was positive in small cell 
A

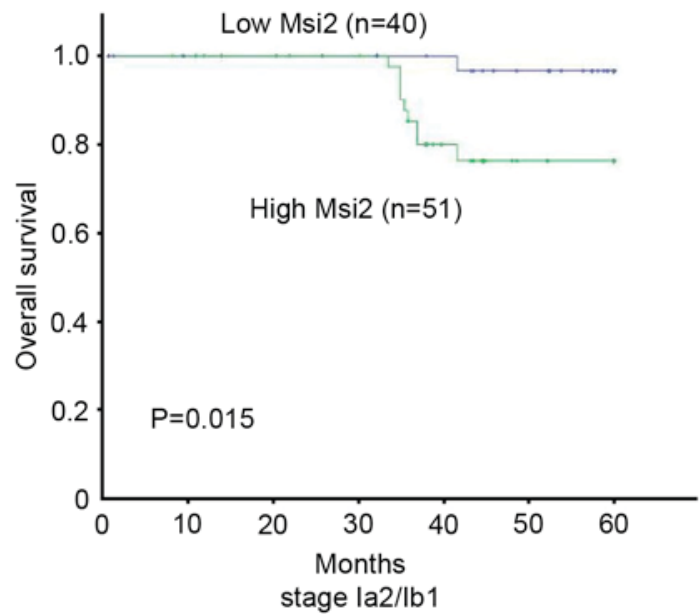

C

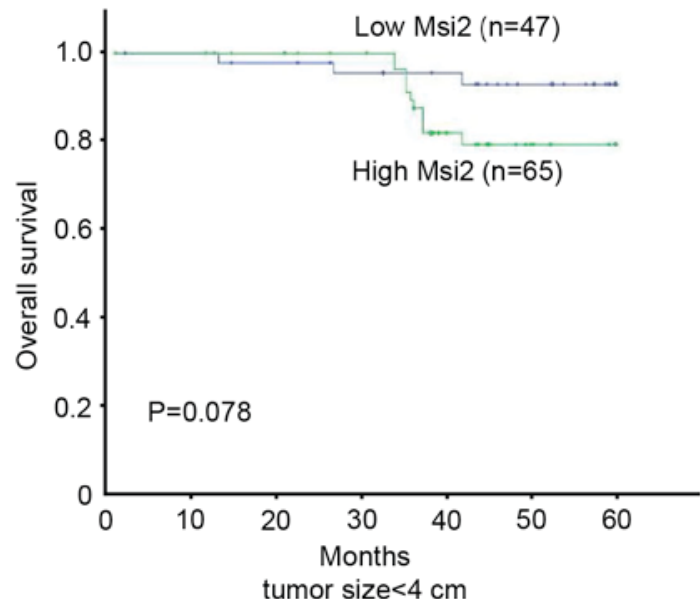

E

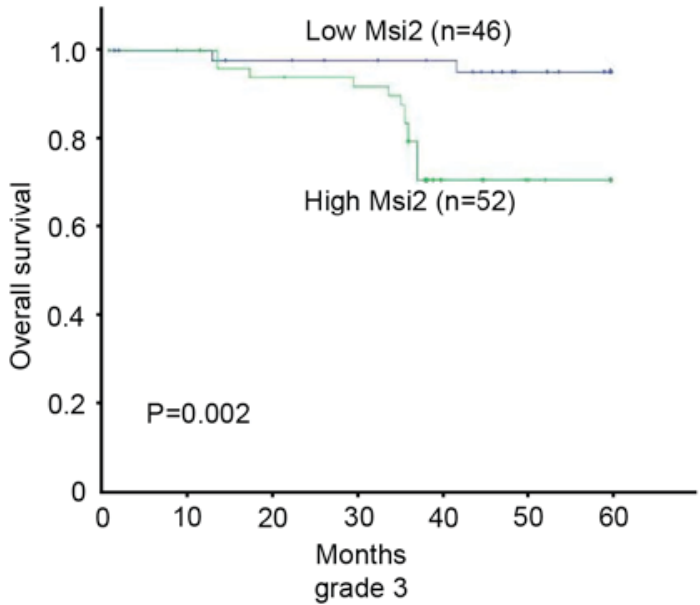

B

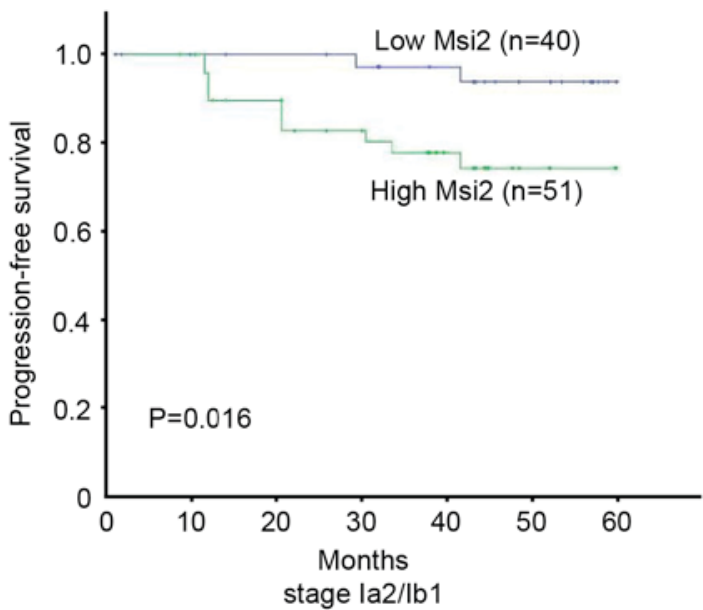

D

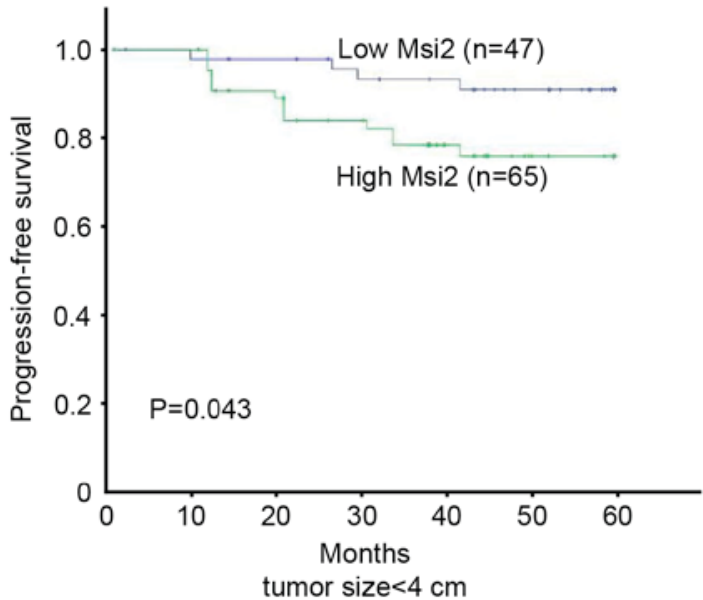

F

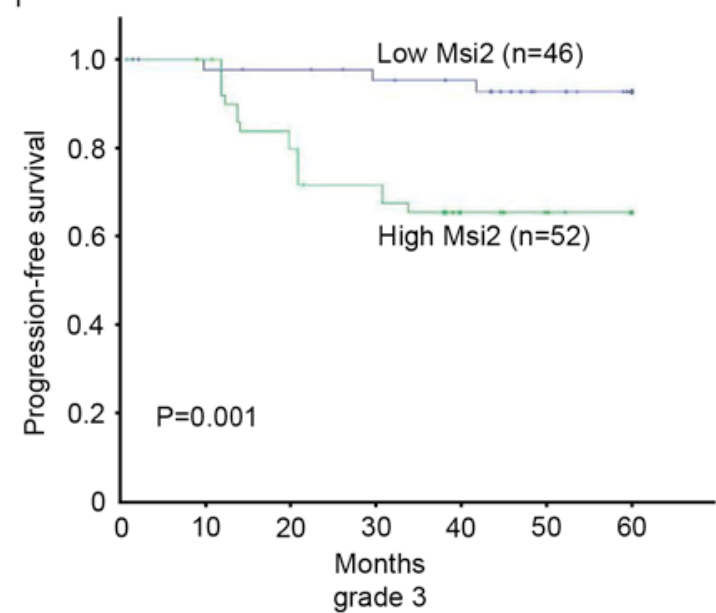

Figure 3. Kaplan-Meier analysis of OS and PFS in relation to Msi2 expression. (A) OS and (B) PFS of the 91 patients with cervical cancer with FIGO stage $\leq$ IB, (C) OS and (D) PFS of the 112 patients with tumor size $\leq 4 \mathrm{~cm}$ and (E) OD and (F) PFS of the 98 patients with grade 3 tumors. FIGO, International Federation of Gynecology and Obstetrics; Msi2, Musashi-2; OS, overall survival; PFS, progression-free survival.

lung carcinomas but was negative in the terminal bronchioles and peritumoral reactive type II pneumocytes of normal lungs. Emadi-Baygi et al (18) demonstrated that the expression of Msi2 mRNA was decreased in grade II compared with grade I gastric cancer tissue; however, Msi2 mRNA expression did not differ significantly between tumor and non-tumor tissues and between different tumor types in gastric cancer. The results of the current study demonstrated that Msi2 expression is upregulated in cervical cancer cells compared with normal cervical cells. Collectively, the results of these studies suggest that the effect of Msi2 may be tissue specific and vary between different tissues.

As an RBP, Msi2 is involved in the regulation of malignant progression and tumor metastasis. He et al (19) indicated that Msi2 promotes the invasion of HCCs by stimulating the 
A
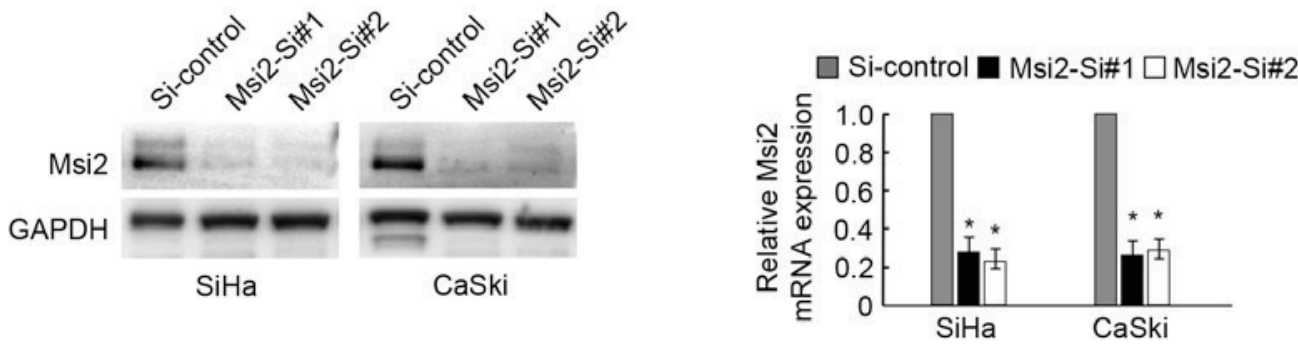

B
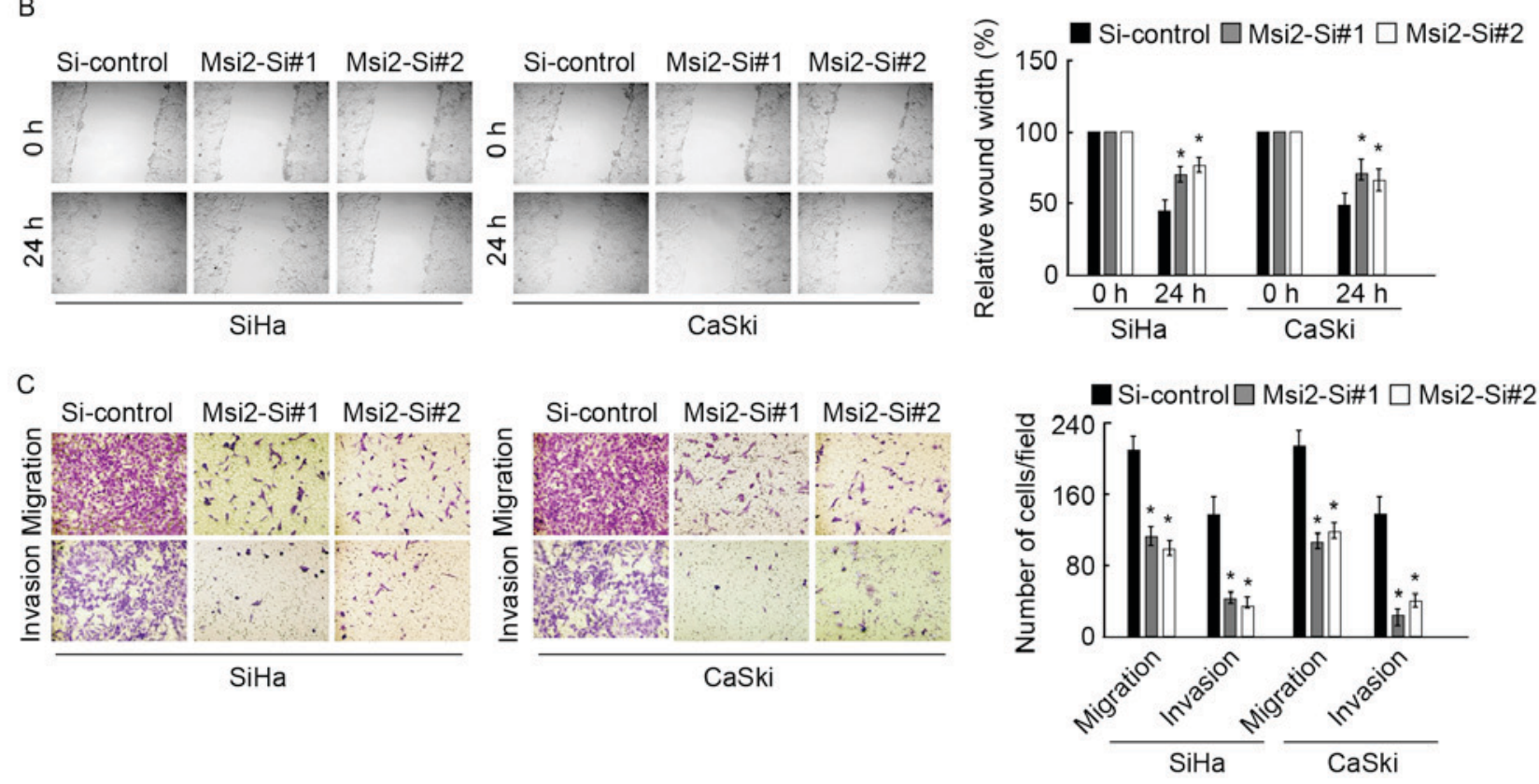

Figure 4. Knockdown of Msi2 inhibits the migration and invasion capacities of cervical cancer cell in vitro. (A) The transfection efficiency of the siRNA was analyzed by measuring the expression of Msi2 mRNA and protein in Sinha and Caskie cells. (B) Effect of Msi2 knockdown in cell migration of Sinha and Caskie was determined by wound healing assay and the uncovered areas in the wound healing assays were quantified as a percentage of the original wound area. (C) Transwell cell migration and Matrigel invasion assays of cervical cancer cells transfected with Msi2-Si\#1, Msi2-Si\#2, and Si-control were performed (magnification, x200). Quantification of cells migrating through the membrane and cells invading through Matrigel in each cell line are presented as proportions of their vector controls. Bar graphs represent the statistical analysis of three independent experiments. "P<0.05 vs. Si-control. Msi2, Msi2, Musashi-2; si, small interfering.

epithelial-mesenchymal transition (EMT), as inhibiting Msi2 expression significantly decreased the invasion of HCC cells and altered the expression of EMT markers. Kaeda et al (20) reported that increased Msi2 expression is associated with more aggressive chronic myeloid leukemia. Likewise, Yang et al (21) indicated that the high expression of Msi2 was associated with disease progression in an independent set of bladder cancer specimens and that the depletion of Msi2 in bladder cancer cells reduced the invasion and metastatic potential of cells. The current study demonstrated that the expression of Msi2 in human cervical tissues was significantly correlated with clinical stage and lymph node metastasis. The results indicated that Msi2 serves an oncogenic role in the progression of malignancies and that knockdown of Msi2 with specific siRNA in Sinha and Caskie cell lines decreases the migration and invasion of cancer cells. Further studies are required to determine the underlying molecular mechanism of the process.

Importantly, the present study demonstrated that Msi2 expression was associated with poor clinical outcomes in patients with cervical cancer. It had been previously demonstrated that Msi2 expression is correlated with the prognosis of patients with hematological malignancies (9). However, few studies had evaluated the prognostic role of Msi2 in solid tumors, apart from in HCC, in which it was suggested that Msi2 promotes the invasion of HCCs by inducing the EMT and thereby contributes to the poor prognosis of patients with HCC (18). Therefore, the current study presents further evidence identifying the correlation between the overexpression of Msi2 and the progression and poor prognosis of patients with solid tumors. In addition, it was determined that high Msi2 expression was predictive of poor overall survival in patients with early stage tumors, small tumor sizes and poorly differentiated tumors, indicating that Msi2 may stimulate the progression of early stage cervical cancer. This hypothesis is supported by Wuebben et al (22), who proposed that Msi2 was associated with several transcription factors during the early stages of embryonic stem cell differentiation.

To conclude, the current study indicated that Msi2 may promote the progression of cervical cancer and that the upregulation of Msi2 expression is associated with the poor prognosis and decreased survival of patients with cervical cancer. Further studies are required to investigate the underlying molecular mechanisms by which Msi2 promotes the development of cervical cancer. 


\section{References}

1. Colombo N, Carinelli S, Colombo A, Marini C, Rollo D and Sessa C; ESMO Guidelines Working Group: Cervical cancer: ESMO Clinical Practice Guidelines for diagnosis, treatment and follow-up. Ann Oncol 23 (Suppl 7): vii27-vii32, 2012.

2. Yeung TL, Leung CS, Yip KP, Au Yeung CL, Wong ST and Mok SC: Cellular and molecular processes in ovarian cancer metastasis. A Review in the Theme: Cell and molecular processes in cancer metastasis. Am J Physiol Cell Physiol 309: C444-C456, 2015.

3. Shi JF, Canfell K, Lew JB and Qiao YL: The burden of cervical cancer in China: Synthesis of the evidence. Int J Cancer 130: 641-652, 2012

4. Warzecha CC, Sato TK, Nabet B, Hogenesch JB and Carstens RP: ESRP1 and ESRP2 are epithelial cell-type-specific regulators of FGFR2 splicing. Mol Cell 33: 591-601, 2009.

5. Degrauwe N, Suva ML, Janiszewska M, Riggi N and Stamenkovic I: IMPs: An RNA-binding protein family that provides a link between stem cell maintenance in normal development and cancer. Genes \& development 30: 2459-2474, 2016.

6. Katz Y, Li F, Lambert NJ, Sokol ES, Tam WL, Cheng AW, Airoldi EM, Lengner CJ, Gupta PB, Yu Z, et al: Musashi proteins are post-transcriptional regulators of the epithelial-luminal cell state. eLife 3: e03915, 2014.

7. Horisawa K, Imai T, Okano $\mathrm{H}$ and Yanagawa $\mathrm{H}$ : The Musashi family RNA-binding proteins in stem cells. Biomolecular concepts 1: 59-66, 2010.

8. Park SM, Deering RP, Lu Y, Tivnan P, Lianoglou S, Al-Shahrour F, Ebert BL, Hacohen N, Leslie C, Daley GQ, et al: Musashi-2 controls cell fate, lineage bias and TGF-beta signaling in HSCs. J Exp Med 211: 71-87, 2014.

9. Hope KJ, Cellot S, Ting SB, MacRae T, Mayotte N, Iscove NN and Sauvageau G: An RNAi screen identifies Msi2 and Prox1 as having opposite roles in the regulation of hematopoietic stem cell activity. Cell Stem Cell 7: 101-113, 2010.

10. Kharas MG, Lengner CJ, Al-Shahrour F, Bullinger L, Ball B, Zaidi S, Morgan K, Tam W, Paktinat M, Okabe R, et al: Musashi-2 regulates normal hematopoiesis and promotes aggressive myeloid leukemia. Nat Med 16: 903-908, 2010.

11. Wang MH, Qin SY, Zhang SG, Li GX, Yu ZH, Wang K, Wang B, Teng MJ and Peng ZH: Musashi-2 promotes hepatitis B virus related hepatocellular carcinoma progression via the Wnt/ß-catenin pathway. Am J Cancer Res 5: 1089-1100, 2015.

12. Barbouti A, Höglund M, Johansson B, Lassen C, Nilsson PG, Hagemeijer A, Mitelman F and Fioretos T: A novel gene, MSI2, encoding a putative RNA-binding protein is recurrently rearranged at disease progression of chronic myeloid leukemia and forms a fusion gene with HOXA9 as a result of the cryptic $t(7 ; 17)$ (p15;q23). Cancer Res 63: 1202-1206, 2003.
13. Kudinov AE, Deneka A, Nikonova AS, Beck TN, Ahn YH, Liu X, Martinez CF, Schultz FA, Reynolds S, Yang DH, et al: Musashi-2 (MSI2) supports TGF- $\beta$ signaling and inhibits claudins to promote non-small cell lung cancer (NSCLC) metastasis. Proc Natl Acad Sci USA 113: 6955-6960, 2016.

14. Pecorelli S: Revised FIGO staging for carcinoma of the vulva, cervix and, endometrium. Int J Gynaecol Obstet 105: 103-104, 2009.

15. Sakakibara S, Nakamura Y, Yoshida T, Shibata S, Koike M, Takano H, Ueda S, Uchiyama Y, Noda T and Okano H: RNA-binding protein Musashi family: Roles for CNS stem cells and a subpopulation of ependymal cells revealed by targeted disruption and antisense ablation. Proc Natl Acad Sci USA 99: 15194-15199, 2002.

16. Livak KJ and Schmittgen TD: Analysis of relative gene expression data using real-time quantitative PCR and the 2(-Delta Delta $\mathrm{C}(\mathrm{T})$ ) method. Methods 25: 402-408, 2001.

17. Moreira AL, Gonen M, Rekhtman N and Downey RJ: Progenitor stem cell marker expression by pulmonary carcinomas. Mod Pathol 23: 889-895, 2010.

18. Emadi-Baygi M, Nikpour P, Mohammad-Hashem F, Maracy MR and Haghjooy-Javanmard S: MSI2 expression is decreased in grade II of gastric carcinoma. Pathol Res Pract 209: 689-691, 2013.

19. He L, Zhou X, Qu C, Hu L, Tang Y, Zhang Q, Liang M and Hong J: Musashi2 predicts poor prognosis and invasion in hepatocellular carcinoma by driving epithelial-mesenchymal transition. J Cell Mol Med 18: 49-58, 2014.

20. Kaeda J, Ringel F, Oberender C, Mills K, Quintarelli C, Pane F, Koschmieder S, Slany R, Schwarzer R, Saglio G, et al: Up-regulated MSI2 is associated with more aggressive chronic myeloid leukemia. Leuk Lymphoma 56: 2105-2113, 2015.

21. Yang C, Zhang W, Wang L, Kazobinka G, Han X, Li B and Hou T: Musashi-2 promotes migration and invasion in bladder cancer via activation of the JAK2/STAT3 pathway. Lab Invest 96: 950-958, 2016.

22. Wuebben EL, Mallanna SK, Cox JL and Rizzino A: Musashi2 is required for the self-renewal and pluripotency of embryonic stem cells. PLoS One 7: e34827, 2012.

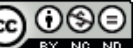

This work is licensed under a Creative Commons Attribution-NonCommercial-NoDerivatives 4.0 International (CC BY-NC-ND 4.0) License. 